\title{
The interaction between massive and massless gravitons by the perturbing topological field theory
}

\author{
E.Koorambas \\ 8A Chatzikosta, 11521 Ampelokipi, Athens, Greece \\ Email: elias.koor@gmail.com
}

August 6, 2011

\begin{abstract}
We test the N.Wu gauge theory of gravity with massive gravitons in the perturbing topological field theory framework. We show that the computation of the correlation function between massive and massless gravitons is reported up to 4-loop and appears to be unaffected by radiative correction. This result ensures the stability of the linking number between massive and massless gravitons with respect to the local perturbation, a result with potential wider applications in cosmology.
\end{abstract}

PACS number(s): 11.15.-q, 04.60.-m, 95.35. +d, 11.10.Gh

Key-Words: gauge field, quantum gravity, dark matter and dark energy, topological field theory

\section{Contents}

2. The interaction between massive and massless gravitons in the topological field theory...... 2

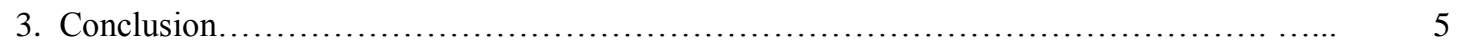

References................................................................. 6

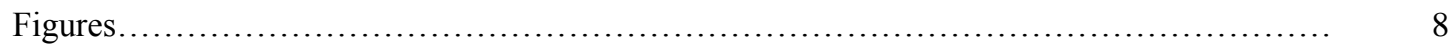

This work is licensed under the Creative Commons Attribution 3.0 Unported License. To view a copy of this license, visit http://creativecommons.org/licenses/by/3.0/ or send a letter to Creative Commons, 444 Castro Street, Suite 900, Mountain View, California, 94041, USA. 


\section{Introduction}

Various authors have attempted to derive General Relativity from a gauge-like principle, involving invariance of physics under transformations of the locally (i.e., in the tangent space at each point) acting Lorentz or Poincar'e group. ([1], [2], [3], [4]).

Gauge theory of gravity is based on the principle of local gauge invariance. Since the model requires strict local gravitational gauge symmetry ([4]), gauge theory is a pertubatively renormalizable quantum model. In the original model, all gauge gravitons are massless ([5]). The massive gravitons were described effectively by Fierz and Pauli ([6]). According to their theory, the existence of massive gravitons would violate the local gauge symmetry of the Lagranian ([7]).

Building on this earlier work, N.Wu proposed a mechanism which introduces massive gravitons without violating the local gauge symmetry of the Lagranian ([4], [8], [9], and [10]).

The third-order gravitational gauge field $C_{3 \mu}^{a}$ is massive if mass is very large. Such a field has no contribution to the long-range gravitational force. Long-range gravitational force results exclusively from the contribution of the fouth-order gravitational gauge field $C_{4 \mu}^{a}$ and obeys inverse square law.

If the mass term of gravitational gauge field is extremely small, however, the third-order gravitational gauge field $C_{3 \mu}^{a}$ will contribute to the middle range gravitational force with approximate range $L \approx \frac{h c}{m}$ (where $\mathrm{h}$ is the Plank constant and $\mathrm{c}$ is the speed of light). For graviton mass $2 \times 10^{-7} \mathrm{eV}$ the gravitational force range will be about one metre.

Recent results from cosmological observation, especially from Cosmic Microwave Background (CMB) temperature anisotropy, suggest that our Universe is essentially flat and that it consists of mainly dark matter and dark energy [11]. A natural origin for dark matter and dark energy is to regard it as consisting of massive gravitons. There are indications that those massive gravitons with mass $2 \times 10^{-7} \mathrm{eV}$ can produce today's acceleration of the Universe ([12], [13]).

In the traditional gauge treatment of gravity the Lorentz group is localized. The gravitational field is, thus, not represented by gauge potential, but by the metric field $\mathrm{g}_{\mu v}$.

Here we propose an alternative understanding of gravity resulting from the extension of N.Wu's gauge theory of gravity with massive gravitons into the framework of perturbing topological field theory. Based on this, we calculate the Feynman diagrams for the interaction between massive and massless gravitons in the topological field theory ([14], [15], [16], [17], [18], [19], [20], [21], and [22]).

These calculations provide insights to interactions between gravity and dark energy. They could, therefore, have important repercussions for current cosmological problems.

2. The interaction between massive and massless gravitons in the topological field theory Taking Wu's gauge model as our starting point ([4], [8], [9], [10], [23], [24], [25]), we introduce two gravitational gauge fields $\left(C_{\mu}^{a}, C_{2 \mu}^{a}\right)$ simultaneously. Since $\left(C_{\mu}^{a}, C_{2 \mu}^{a}\right)$ are vectors in Lie algebra ([8], [9]), they can be expanded as

$C_{\mu}=C_{\mu}^{a} \hat{P}_{a} \quad, C_{2 \mu}=C_{2 \mu}^{a} \hat{P}_{a}$ 
These correspond with two gauge covariant derivatives

$D_{\mu}=\partial_{\mu}-i g C_{\mu}(x) \quad, D_{2 \mu}=\partial_{\mu}+i a g C_{2 \mu}(x)$

and two different field strengths, given by

$$
\begin{aligned}
& F_{\mu v}=\frac{1}{-i g}\left[D_{\mu}, D_{v}\right] \\
& F_{2 \mu v}=\frac{1}{i a}\left[D_{2 \mu}, D_{2 v}\right]
\end{aligned}
$$

The Lagrangian of the system is given by

$$
\begin{aligned}
& \mathfrak{\Im}_{0}=-\frac{1}{4} \eta^{\mu \rho} \eta^{v \sigma} g_{\beta \gamma} F_{\mu \nu}^{\beta} F_{\rho \sigma}^{\gamma}-\frac{1}{4} \eta^{\mu \rho} \eta^{v \sigma} g_{\beta \gamma} F_{2 \mu \nu}^{\beta} F_{2 \rho \sigma}^{\gamma} \\
& -\frac{m^{2}}{2\left(1+a^{2}\right)} \eta^{\mu \nu} g_{\beta \gamma}\left(C_{\mu}^{\beta}+a C_{2 \mu}^{\beta}\right)\left(C_{v}^{\gamma}+a C_{2 v}^{\gamma}\right)
\end{aligned}
$$

where $m$ is the constant mass parameter. The action of the system is given by

$$
S=\int d^{4} x J(C) \mathfrak{I}_{0}
$$

Equation (4) gives the mass term in gravitational gauge fields. To obtain the Eigenstates of mass matrix the following rotation is needed

$$
\begin{aligned}
& C_{3 \mu}=\cos \theta C_{\mu}+\sin \theta C_{2 \mu} \\
& C_{4 \mu}=-\cos \theta C_{\mu}+\sin \theta C_{2 \mu}
\end{aligned}
$$

where the angle $\theta$ is given by

$$
\cos \theta=\frac{1}{\sqrt{1+a^{2}}}, \quad \sin \theta=\frac{a}{\sqrt{1+a^{2}}}
$$

After transformation (6), the Lagrangian of the system is given by

$$
\mathfrak{I}_{0}=-\frac{1}{4} \eta^{\mu \rho} \eta^{\nu \sigma} g_{\beta \gamma} F_{30 \mu \nu}^{\beta} F_{30 \rho \sigma}^{\gamma}-\frac{1}{4} \eta^{\mu \rho} \eta^{\nu \sigma} g_{\beta \gamma} F_{40 \mu \nu}^{\beta} F_{40 \rho \sigma}^{\gamma}
$$

where $F_{30 \mu v}^{a} F_{40 \mu v}^{a}$ are given by: 


$$
\begin{aligned}
& F_{30 \mu \nu}^{a}=\partial_{\mu} C_{3 v}^{a}-\partial_{\nu} C_{3 \mu}^{a} \\
& F_{40 \mu \nu}^{a}=\partial_{\mu} C_{4 \nu}^{a}-\partial_{\nu} C_{4 \mu}^{a}
\end{aligned}
$$

From the above follows that the three-dimensional gravitational gauge field $C_{3 \mu}^{a}$ is massive, with mass $m$, whereas the four-dimensional gravitational gauge field $C_{4 \mu}^{a}$, is massless.

To investigate the interaction between the massive gravitational gauge field $C_{3 \mu}^{a}$ and the massless gravitational gauge field $C_{4 \mu}^{a}$ we use topological field theory.

Our computation of the correlation function between the massive and massless gravitons is analogous with that given in the literature ([22]).

$$
\left\langle\int_{\gamma_{2}} d y^{v} C_{4 \mu}^{a} \int_{\gamma_{1}} d x^{\mu} C_{3 \mu}^{\beta}\right\rangle_{S_{e f f}}
$$

For the case of the two gravitational gauge fields $C_{3 \mu}^{a}$ and $C_{4 \mu}^{a}$ lying on two smooth, closed, non-intersecting curves $\gamma_{1}$ and $\gamma_{2}$ (Fig.1), the computation of the correlation function is reported up to 4- loop. This computation shows that the correlation function is unaffected by radiative correction. This result ensures the stability of the linking number with respect to the local perturbation ([22]). The local perturbation can be added to the Chern - Simons action given by

$$
S_{\text {eff }}=\frac{1}{2} \int d^{3} x \varepsilon^{\mu v \rho} C_{4 \mu}^{a} \partial_{\nu} C_{3 \rho}^{\gamma}+\frac{\tau}{2 !} \int d^{3} x \tilde{F}_{a}^{4 \mu} \tilde{F}_{4 \mu}^{a} \tilde{F}_{\beta}^{3 v} \tilde{F}_{3 v}^{a}([22])
$$

with $\tilde{F}_{\alpha}^{4 \mu}=\frac{1}{2} \varepsilon^{4 \mu 3 v \rho} F_{\alpha 3 v \rho}$ and $\tau$ being an arbitrary parameter with negative mass dimension, reflecting the power-counting non-renormalizability of the perturbation.

The Feynman diagrams for the interaction between massive and massless gravitons in the topological field theory are similar to those described at [22]. To calculate the correlator function (10) we use the action

$$
S_{\text {eff }}=\frac{1}{2} \int d^{3} x \varepsilon^{\mu v \rho} C_{4 \mu}^{a} \partial_{\nu} C_{3 \rho}^{\gamma}+\int d^{3} x b \partial C_{3 \rho}^{\gamma}+\frac{\tau}{4 !} \int d^{3} x: \tilde{F}_{a}^{4 \mu} \tilde{F}_{4 \mu}^{a} \tilde{F}_{\beta}^{3 v} \tilde{F}_{3 v}^{\beta}:, \ldots \ldots
$$

The Lagrange multiple $b$ is introduced to this function to implement the Landau gauge. To evaluate the Feynman diagrams we use the following equations: 


$$
\begin{aligned}
& \partial^{2} \frac{1}{|x-y|}=-4 \pi \delta^{3}(x-y) \\
& \left\langle C_{4 \mu}^{a}(x), C_{3 v}^{\beta}(y)\right\rangle_{S_{e f f}}=\frac{g^{\alpha \beta}}{4 \pi} \varepsilon_{\mu v \rho} \frac{(x-y)^{\rho}}{|x-y|^{3}} \\
& \left\langle C_{4 \mu}^{a}(x), \bar{F}_{3 v}^{\beta}(y)\right\rangle=g^{\alpha \beta} g_{\mu \nu} \delta(x-y)+\partial_{\mu} \partial_{v} \frac{1}{4 \pi|x-y|} \\
& \left\langle\bar{F}_{4 v}^{a}(x), \bar{F}_{3 v}^{\beta}(y)\right\rangle=-g^{\alpha \beta} \varepsilon_{\mu v \rho} \partial^{\mu} \delta^{3}(x-y)
\end{aligned}
$$

The Feynman diagrams that contribute to the correlation function (10) are of two-loop order (see Figure 2). Based on [22], it follows that, for the second-, third- and fourth-order diagrams (see Figures 2, 3, 4), and more generally, for all terms in all possible diagrams between massive and massless gravitons, these Feynman diagrams are proportional to $\delta^{3}(x-y)$ or its derivatives ([22]).

$$
\begin{aligned}
& I^{(2)}=4 \int_{\gamma_{1}} d x^{\mu} \int_{\gamma_{2}} d y^{v} \int d^{3} z_{1} d^{3} z_{2} \delta^{3}\left(x-z_{1}\right) \delta^{3}\left(y-z_{2}\right) \\
& \times\left[\varepsilon_{\beta \rho \lambda} \partial^{\lambda} \delta^{3}\left(z_{1}-z_{2}\right)\right]\left[\varepsilon_{\mu}^{\rho \tau} \partial_{\tau} \delta^{3}\left(z_{1}-z_{2}\right)\right] \\
& \times\left[\varepsilon^{\beta \sigma} \partial_{\sigma} \delta^{3}\left(z_{1}-z_{2}\right)\right] \\
& I^{(3)}=\int_{\gamma_{1}} d x^{\mu} \int_{\gamma_{2}} d y^{v} \int d^{3} z_{3}\left[\tilde{\partial}_{\mu \nu} \delta^{3}(x-y)\right]\left[\tilde{\partial}_{\beta \delta} \delta_{\varepsilon}\left(x-z_{3}\right)\right]\left[\tilde{\partial}^{\beta \lambda} \delta^{3}\left(x-z_{3}\right)\right] \\
& {\left[\tilde{\partial}_{\rho \lambda} \delta_{\varepsilon}\left(y-z_{3}\right)\right]\left[\tilde{\partial}^{\rho \delta} \delta^{3}\left(y-z_{3}\right)\right]} \\
& I^{(4)}=\int_{\gamma_{1}} d x^{\mu} \int_{\gamma_{2}} d y^{v} \int d^{3} z_{3} d^{3} z_{4}\left[\tilde{\partial}_{\mu \nu} \delta^{3}(x-y)\right]\left[\tilde{\partial}^{\beta \lambda} \delta_{\varepsilon}\left(x-z_{3}\right)\right]\left[\tilde{\partial}_{\beta \sigma} \delta^{3}\left(x-z_{4}\right)\right] \\
& {\left[\tilde{\partial}^{\rho \sigma} \delta^{3}\left(y-z_{3}\right)\right]\left[\tilde{\partial}_{\rho \lambda} \delta^{3}\left(y-z_{3}\right)\right]\left[\tilde{\partial}^{\varphi \omega} \delta_{\varepsilon}\left(z_{3}-z_{4}\right)\right]\left[\tilde{\partial}_{\varphi \omega} \delta^{3}\left(z_{3}-z_{4}\right)\right]}
\end{aligned}
$$

Since it is always $x \neq y$, the second-, third- and fourth-order diagrams correspond to null correction to the basic diagrams. The correlator function (10), therefore, is the link number to all orders:

$$
\left\langle C_{3 \mu}^{\beta}(x), C_{4 v}^{a}(y)\right\rangle_{S_{e f f}}=\chi\left(\gamma_{1}, \gamma_{2}\right)
$$

\section{Conclusion}

Equation (18) links massive graviton, $C_{3 \mu}^{a}$ representing dark energy, with the massless graviton, $C_{4 \mu}^{a}$ representing the gravity. This expression of the interaction between gravity and dark energy may have potentially very important implications to cosmology. We find that the interactions between dark energy and gravitons are independent of metric. These interactions are, therefore, within the framework of perturbing topological field theory. The topological properties of these interactions are represented by knots and links. The size, exact shape, location etc of these knots and links are not of immediate concern for the problem at hand (interaction between dark mater/energy and gravitons). 
Equation (18), that links massive and massless gravitons, is independent of the exact location, size and shape of the two knots. Equation (18) depends only on the topological relationship of the knots with each other. This invariant may have a physical interpretation: it may represent the work done to move a massive graviton (dark energy) around one knot in three dimensional space while a massless graviton runs around the other knot.

\section{References}

[1].Utiyama, R. 1956 Invariant theoretical interpretation of interaction. Physical Review101 1597-1607.

[2].Kibble, T W.B. 1961. Lorentz invariance and the gravitational field. Journal of Mathe matical Physics 2 212-221.

[3].Wilczek, F. 1998. Riemann-Einstein structure from volume and gauge symmetry. Physical

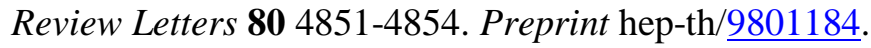

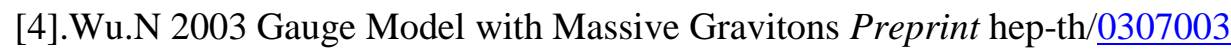

[5]. Blagojevic.M 2002 Gravitation and gauge symmetries I.O.P Publishing Ltd pp $214-219$

[6].Fierz M and Pauli W 1939 Relativistic wave equation for particles of arbitrary spin in an electromagnetic field Proc. R. Soc. A 173211

[7].S. Dubovsky.S, Tkachev.P.T.I 2004 Preprint hep-th/ㅁ11158

[8].Wu.N Quantum Gauge Theory of Gravity” 2002 Devision of Particles and Fields Aps Williamsburg, Virgia USA

[9].Wu.N Gauge Theory of Gravity 2001 Preprint hep-th/ㅁ109145

[10].Wu.N Renormalizable Quantum Gauge Theory of Gravity 2002 Preprint hep-th/0207254

[11].Hu.W, Dodelson.S 2002Annu.Rev.Astron.Astrophys 40 1-51

[12].Kiritsis.E 2006 Preprint hep-th/ $\underline{0608088}$

[13].Aharony.O, Clark.A, Karch.A 2006 Preprint hep-th/ㅇ6요

[14].Atiyah.M 1989 The Geometry and Physics of Knots, Cambridge Univ. Press

[15].Witten.E 1989 Commun. Math. Phys. 121 351-399

[16].Schwarz.A.S 1987 New topological invariants in the theory of quantized fields, Baku International Conference

[17].Kaul.R.K 1992 Complete solution of SU (2) Chern-Simons theory Preprint hepth/ $\underline{\underline{212129}}$

[18].Kaul.R.K 1994 Commun. Math. Phys. 162289

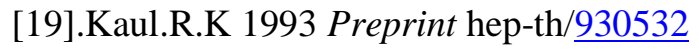


[20].Kaul.R.K 1998 Chern-Simons theory, knot invariants, vertex models and three-manifold invariants Preprint hep-th/ $\underline{9804122}$

[21].Kaul.R.K 1999 Topological Quantum Field Theories -A Meeting Ground for Physicists and Mathematicians Preprint hep-th/9907119

[22].Vitor E, R.Lemes, Cesar .A 1999 A perturbing topological field theories Phys. Rev D 60 065008

[23]. Wu.N 2001Commun.Theor.Phys 36169

[24]. Wu.N 2002Commun.Theor.Phys 38 577-582

[25]. Wu.N 2002Commun.Theor.Phys 38 151-156

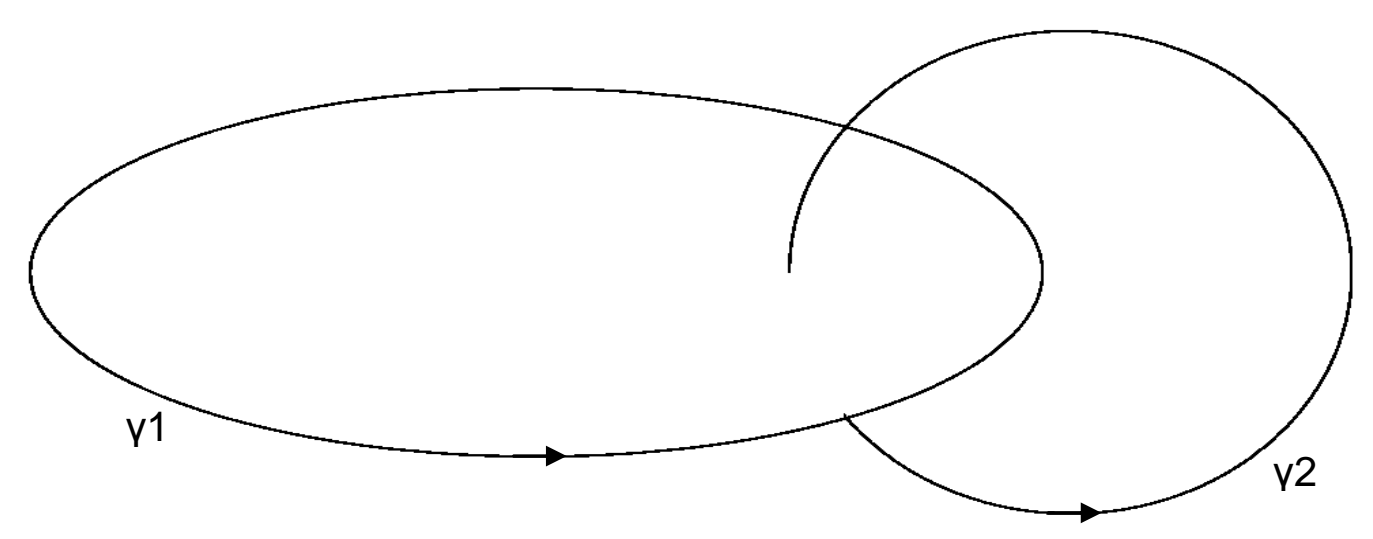

Fig.1 Linking between $\gamma_{1}, \gamma_{2}$

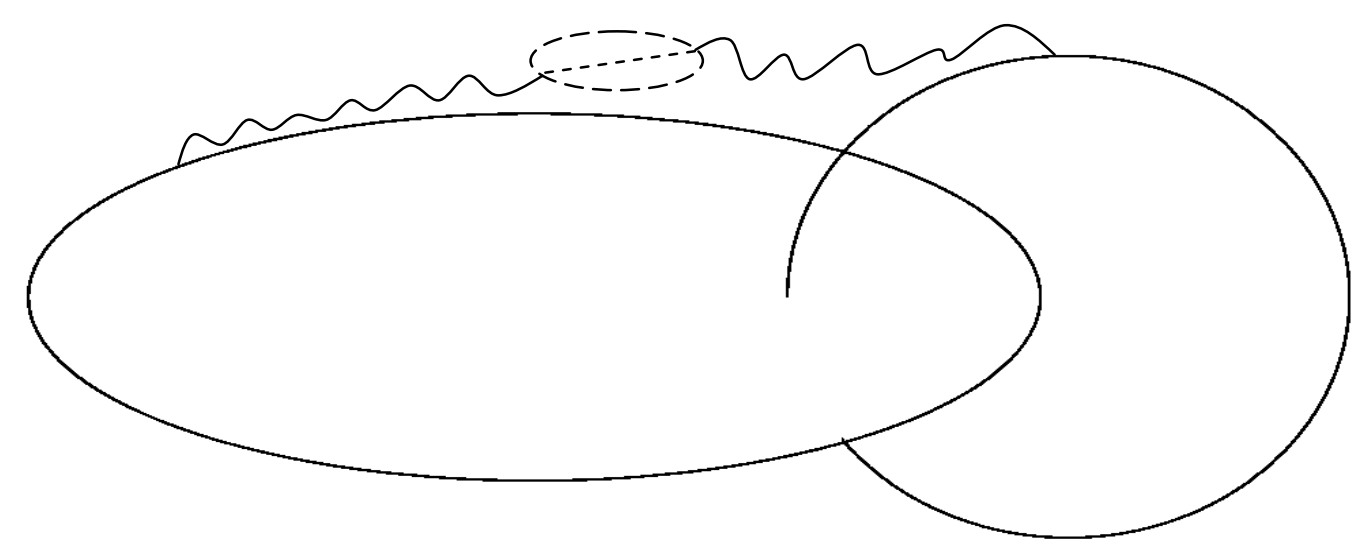

Fig.2 Two loop contribution. 

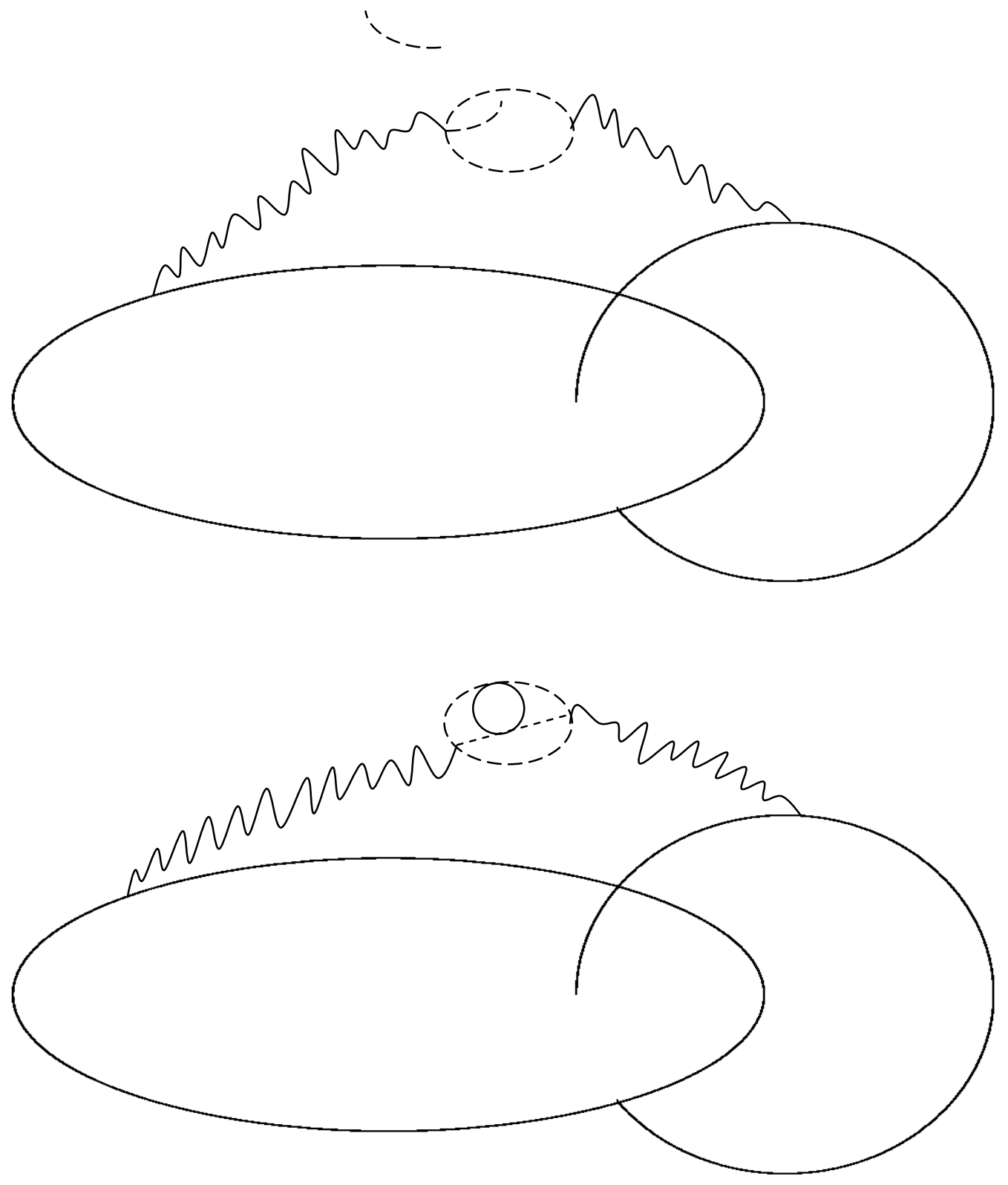

Fig.3 Three loop contribution.Fig. 4 Four loop contribution. 